\title{
Interacting models may be key to solve the cosmic coincidence problem
}

\author{
Sergio del Campo*, ${ }_{1}^{1}$ Ramón Herrera ${ }^{\dagger},{ }^{1}$ and Diego Pavón ${ }^{\ddagger 2}$ \\ ${ }^{1}$ Instituto de Física, Pontificia Universidad Católica de Valparaiso, \\ Avenida Brasil 2950, Casilla 4059, Valparaíso, Chile \\ ${ }^{2}$ Departamento de Física, Facultad de Ciencias, \\ Universidad Autónoma de Barcelona, \\ 08193 Bellaterra (Barcelona), Spain
}

\begin{abstract}
It is argued that cosmological models that feature a flow of energy from dark energy to dark matter may solve the coincidence problem of late acceleration (i.e., "why the energy densities of both components are of the same order precisely today?"). However, much refined and abundant observational data of the redshift evolution of the Hubble factor are needed to ascertain whether they can do the job.
\end{abstract}

\footnotetext{
* Electronic Mail-address: sdelcamp@ucv.cl

$\dagger$ E-mail address: ramon.herrera@ucv.cl

$\ddagger$ E-mail address: diego.pavon@uab.es
} 


\section{INTRODUCTION}

As is well known, the popular vacuum-cold dark matter $(\Lambda \mathrm{CDM})$ model fits rather well most observational data, however it suffers from two main -and lasting- shortcomings, namely: the low value of the vacuum energy (about 123 magnitude orders below the quantum field theory estimation) and the coincidence problem. The latter refers to the fact that despite the vacuum energy does not vary with time and dark matter energy depends on redshift as $(1+z)^{3}$ both energy densities are nowadays of the same order. This is why very often the vacuum component is replaced by an evolving dark energy field -see [1, 2] for recent reviews. Nevertheless, this does not satisfactorily solve the problem since the initial value of the dark energy ought to be fine tuned up to obtain the desired result; it only substitutes a fine tuning by another [2].

An encouraging approach that is receiving increasing attention [3] rests on the assumption that both components do not evolve separately but interact with each other, i.e.,

$$
\dot{\rho}_{m}+3 H \rho_{m}=Q, \quad \text { and } \quad \dot{\rho}_{\phi}+3 H\left(\rho_{\phi}+P_{\phi}\right)=-Q
$$

Here, $Q$ stands for the interaction term, and the subscripts $m$ and $\phi$ denote non-relativistic (pressureless) dark matter (DM) and dark energy (DE), respectively. Clearly, this represents a step forward because for $Q>0$ (i.e., for energy transfers from DE to DM) the ratio between both energies, $r \equiv \rho_{m} / \rho_{\phi}$, evolves more slowly than in the $\Lambda$ CDM model. Effectively, in the latter, $\dot{r}=-3 H r$ while in the interacting case its time evolution of governed by

$$
\dot{r}=3 H r\left[w+\frac{\kappa^{2} Q}{9 H^{3}} \frac{(r+1)^{2}}{r}\right],
$$

where $w \equiv P_{\phi} / \rho_{\phi}<-1 / 3$ denotes the equation of state parameter of DE. Equation (2) follows from (11) and Friedmann's equation for spatially flat universes

$$
3 H^{2}=\kappa^{2}\left(\rho_{m}+\rho_{\phi}\right) \quad\left(\kappa^{2} \equiv 8 \pi G\right) .
$$

As we will see below, for reasonable and suitably chosen interaction terms, $r$ either tends to a constant or varies more slowly than the scale factor, $a(t)$, at late times. This certainly alleviates the coincidence problem. It is worthy of note that if $Q$ were negative (signifying an energy transfer from DM to DE) the problem would only worsen, moreover at early times the energy density of DE would have been negative, and, finally, the second law 
of thermodynamics could be violated [4, 5]. Except in chamaleon models [6], baryons as well as radiation usually do not participate in the interaction. The former because of the tight constraints imposed by local gravity measurements [7, 8], the latter because otherwise the photons would not longer follow geodesics which would affect precise measurements of deviations of radar signals grazing the sun.

It is not the aim of this article to solve the coincidence problem -we believe it cannot be done at present- but to devise a simple strategy to do it, within general relativity, as soon as sufficiently accurate and abundant data on the evolution of the Hubble factor becomes available. This paper extends and generalizes a previous work of the authors [9].

Most studies of late acceleration implicitly assume that matter and dark energy interact gravitationally only. In the absence of an underlying symmetry that would suppress a matter-dark energy coupling (or interaction) there is no a priori reason to discard it. Further, the coupling is not only likely but inevitable [10] and its introduction is less arbitrary than otherwise. In fact, the accretion of DE by black holes [11, 12] implies a non-vanishing and positive $Q$. While its small value modifies only very little the dependence of the energy densities on redshift (and cannot substantially alleviate the coincidence problem), it sets a minimum value for the interaction.

The interaction may bring some further consequences. It can push the beginning of accelerated expansion era to higher redshifts. It can also erroneously suggest an equation of state for the dark energy of phantom type (i.e., $w<-1$ ), see [13] and references therein. Moreover, the interaction may give rise to fluctuations in the count of galaxy clusters with redshift [14]. Likewise, the coupling can alter the isothermal Maxwell-Boltzmann velocity distribution of weakly interacting massive particles in the galaxy halos [15] whereby the average dark matter velocity is expected to increase significantly. Since the detection rates of the different experiments searching for dark matter strongly depend on this velocity it is crucial not to dismiss a priori the coupling [16].

Ultimately, the existence or non-existence of the interaction is to be discerned observationally. Whereas the current empirical data are insufficient to settle this issue, a couple of studies seem to favor the former possibility: $(i)$ As it should be expected, the interaction alters the time required for a self-gravitating, collapsing, structure to reach equilibrium as well as the equilibrium configuration itself. Therefore the Layzer-Irvine equation [17, 18] is to be generalized to take into account the interaction. In this regard, from the analysis of 
the dynamics of 33 relaxed galaxy clusters -for which reliable x-ray, weak lensing and optical data are available-, it has been reported a small but not vanishing interaction [19, 20]. (ii) Since the interaction modifies the evolution rate of the metric potentials the integrated Sachs-Wolfe component of the cosmic microwave background radiation (CMB) is enhanced. The cross-correlation of galaxy catalogs with CMB maps also hints at a small interaction [21].

This paper is organized as follows. Next section introduces three different expressions for $Q$ and show that all three lead to a late, everlasting, era of either constant or quasi-constant $r$ regardless of whether $w$ is lower or higher than -1 . Section 3 shows the consistency of the three interacting models with the recent observational bounds of Daly et al. [22] based on data from supernovae type Ia, taken from different sources, and 30 radio-galaxies. The only cosmological assumption behind these bounds is that the Universe can be considered homogeneous and isotropic at large scales. Section 4 devises a simple strategy to solve the coincidence problem. Finally, Section 5 summarizes and discusses our results.

We focus our attention on spatially flat Friedmann-Robertson-Walker models. As usual, a subscript zero means that the corresponding quantity is to be evaluated at present time.

\section{THREE CHOICES FOR THE INTERACTION TERM}

Because of the unknown nature of both DM and DE it does not seem feasible nowadays to derive an expression for $Q$ on first principles. The alternative is to propose phenomenological expressions based on arguments of simplicity and reasonableness. Firstly, note that $Q$ must be small and positive -otherwise the DE could not dominate the expansion today. On the other hand, if it were large and negative, DE would have dominated the expansion practically from the outset and galaxies couldn't have condensed.

Looking at the left hand side of Eqs. (11) it becomes apparent that $Q$ must be a function of the energy densities multiplied by a quantity with units of inverse of time. For the latter the obvious choice is the Hubble factor, so $Q=Q\left(H \rho_{m}, H \rho_{\phi}\right)$. By power law expanding this function and keeping just the first term we get $Q \simeq \lambda_{m} H \rho_{m}+\lambda_{x} H \rho_{x}$. In view of the lack of information about the coupling it is advisable to retain just one parameter (the less observational parameters when fitting to observational data, the better). Then, three

choices follow, namely: $\lambda_{m}=\lambda_{x}, \lambda_{x}=0$, and $\lambda_{m}=0$. Other choices, as $Q \propto \rho_{m} \rho_{x}$ [23], are 
also possible but we will not deal with them here.

We will therefore consider the following three expressions for the coupling,

$$
Q=3 \alpha H\left(\rho_{m}+\rho_{\phi}\right), \quad Q=3 \beta H \rho_{m}, \quad \text { and } \quad Q=3 \eta H \rho_{\phi},
$$

where the phenomenological parameters, $\alpha, \beta$, and $\eta$, are dimensionless, positive-definite small constants. Henceforward, we will call the corresponding models alpha, beta, and eta, respectively.

Model alpha fits very well data from SN Ia, CMB, and large scale structure formation provided $\alpha<2.3 \times 10^{-3}$ [21, 24, 25]. Further, this model possesses the remarkable property that the ratio $r$ tends to a stationary but unstable value at early times, $r_{s}^{+}$, and to a stationary and stable value, $r_{s}^{-}$, (an attractor) at late times. This follows from inserting the corresponding expression for $Q$ in the right hand side of Eq. (2) and setting $\dot{r}$ to zero. For $w=$ constant the stationary solutions of the resulting quadratic equation are:

$$
r_{s}^{ \pm}=-1+2 b \pm 2 \sqrt{b(b-1)}, \quad b=-\frac{w}{4 \alpha}>1 .
$$

As it can be checked by slightly perturbing the right hand side of Eq.(2), the stationary solution $r_{s}^{+}$results unstable while $r_{s}^{-}$is stable [26, 27]. The general solution of Eq. (2)

$$
r(x)=\frac{r_{s}^{-}+x r_{s}^{+}}{1+x}
$$

interpolates between $r_{s}^{+}$and $r_{s}^{-}$. Here, $x=\left(a / a_{*}\right)^{-\mu}$, with $\mu \equiv 12 \alpha \sqrt{b(b-1)}$, and $a_{*}$ denotes the scale factor at which $r$ takes the arithmetic medium value $\left(r_{s}^{+}+r_{s}^{-}\right) / 2$. In the range $r_{s}^{-}<r<r_{s}^{+}$the function $r(x)$ decreases monotonously. Consequently, as the Universe expands, $r(x)$ smoothly evolves from $r_{s}^{+}$to the attractor solution $r_{s}^{-}$. The transition from one asymptotic solution to the other occurred only recently -see left panels of Figs. 1 and 2. so one can take $r \simeq r_{s}^{+}$during a fairly large part of the history of the Universe. Finally, the constraint $r_{s}^{+} \simeq$ constant implies that $r$ and $\alpha$ are not independent, but verify the relationship $\alpha\left(r_{s}^{+}+1\right)^{2}=|w| r_{s}^{+}$, so the product $\alpha r_{s}^{+} \sim|w|$ is of order unity.

As indicated above, this choice of $Q$ yields a constant but unstable ratio at early times and constant and stable lower ratio at late times -left panels of Figs. 1 and 2, It is hard to imagine a simpler expression for the coupling entailing these two properties. Likewise, the aforesaid expression can be re-interpreted as implying an effective exponential potential for the dark energy field at late times and a power-law at early times [24, 26]. 

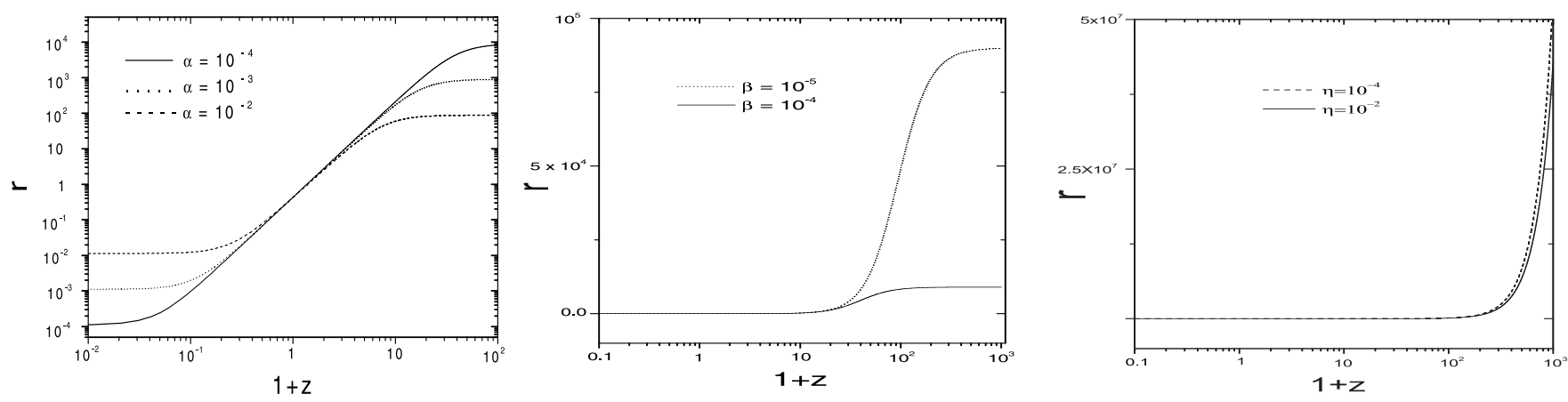

FIG. 1: From left to right, evolution of the ratio $r=\rho_{m} / \rho_{\phi}$ with redshift for models alpha, beta, and eta. For all of them $r$ either tends to a constant or varies very slowly at small redshift. In drawing the curves we have fixed $r_{0}=3 / 7$ and $w=-0.9$.
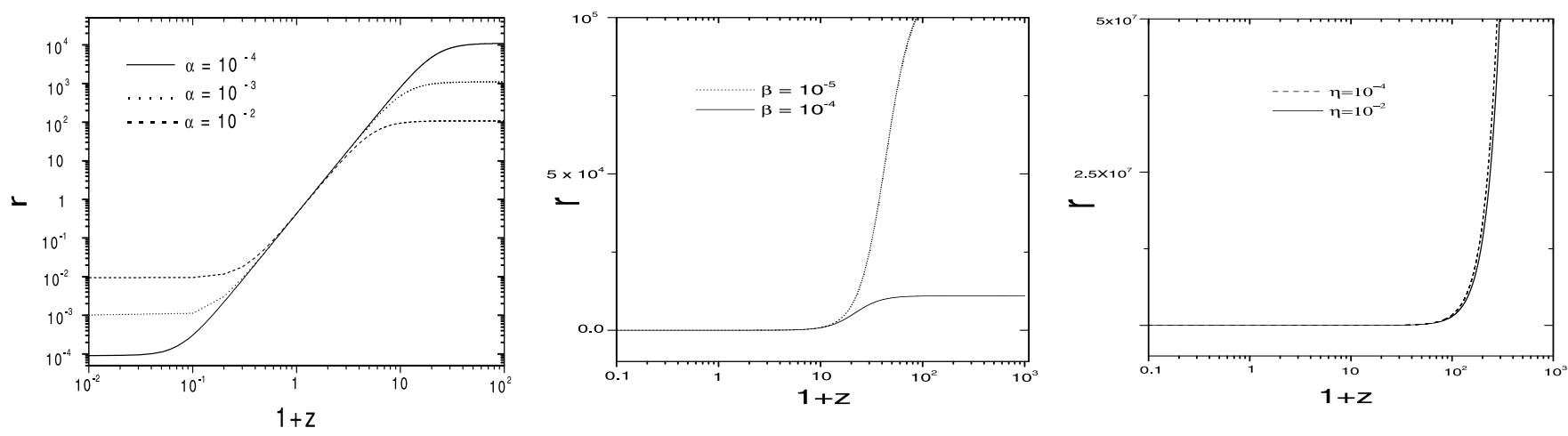

FIG. 2: Same as Fig.1 except for that in this case $w=-1.1$.

Near $r \approx r_{s}^{-}$the balance Eqs. (1) can be approximated by

$$
\begin{aligned}
\frac{1}{\rho_{m}} \frac{d \rho_{m}}{d r} & \simeq \frac{1-\alpha\left(1+1 / r_{s}^{-}\right)}{\alpha\left(r_{s}^{+}-r_{s}^{-}\right)\left(r-r_{s}^{-}\right)}, \\
\frac{1}{\rho_{\phi}} \frac{d \rho_{\phi}}{d r} & \simeq \frac{1+w+\alpha\left(1+r_{s}^{-}\right.}{\alpha\left(r_{s}^{+}-r_{s}^{-}\right)\left(r-r_{s}^{-}\right)} .
\end{aligned}
$$

For $w \simeq$ constant, these equations can be integrated to

$$
\rho_{m} \propto a^{-3\left[1-\alpha\left(1+1 / r_{s}^{-}\right)\right]}, \quad \rho_{\phi} \propto a^{-3\left[1+w+\alpha\left(1+r_{s}^{-}\right)\right]} .
$$

Notice that the condition $\left.\dot{r}\right|_{r=r_{s}^{-}}=0$ implies that the exponents in the energy densities, Eq. (8), coincide. Interestingly, these results are not only valid when the dark energy is 
a quintessence field (i.e., $-1<w<-1 / 3$ ), they also apply when the dark energy is of phantom type (i.e., $w<-1$ ), either a scalar field with the "wrong sign" for the kinetic energy term, a $k$-essence field, or a tachyon field [28].

In the vicinity of the attractor, dark matter and dark energy dominate the expansion whence the scale factor can be written as

$$
a \propto t^{(2 / 3)\left[1+w+\alpha\left(1+r_{s}^{-}\right)\right]^{-1}}
$$

It is easy to show that, at early times, $r^{2}+\left(w \alpha^{-1}+2\right) r+1=0$ and the constant ratio is $r_{s}^{+}=r(z \rightarrow \infty) \sim \alpha^{-1}$ while, at late times, $r_{s}^{-}=r(z \rightarrow-1) \sim \alpha$ [27]. These results mean a conceptual advance with respect to the $\Lambda \mathrm{CDM}$ model. If the present acceleration of the Universe is generated by a cosmological constant, then the initial values of dark matter and dark energy have to be fine-tuned by 96 orders of magnitude at Planck time, i.e., $r\left(t_{\text {Planck }}\right)=\Omega_{m} / \Omega_{\Lambda} \sim \mathcal{O}\left(10^{96}\right)$. In the model of above this ratio is fixed by $\alpha$. Thus, the initial condition problem is significantly alleviated. For example, if $\alpha \sim 10^{-4}$, the initial dark matter to dark energy ratio comes to be $r\left(t_{\text {Planck }}\right) \sim \mathcal{O}\left(10^{4}\right)$.

Model beta does not share the nice feature of a late attractor. However, in this case, for constant $w$ one has [29]

$$
r=\frac{\xi}{\beta r_{0}-(1+z)^{-3 \xi}\left[\beta\left(1+r_{0}\right)+w\right]} r_{0},
$$

where $\xi=-w-\beta>0$. Hence, the evolution of $r$ is slower than in the $\Lambda$ CDM model. There is a monotonous decrease of $r$ from values higher than unity at early times to a nearly constant, value at present time (fixed as 3/7) irrespective of whether dark energy is a quintessence field (middle panel of Fig. 1) or a phantom field (middle panel of Fig. 2). For vanishing scale factor $r$ tends to the finite, constant value $\xi / \beta$ (however, the model should not be extrapolated to such an early stage). Thus, in spite of the absence of a late time attractor this choice of $Q$ also significantly alleviates the coincidence problem as the energy density ratio varies very slowly at present time, i.e., $\left|(\dot{r} / r)_{0}\right| \leq H_{0}$. This feature is shared by model eta -see right panel in Figs. 1 and 2 .

Altogether, all three models considerably alleviate the coincidence problem but there is no guarantee that any of them solves it in full. A successful model should also predict $r_{0} \sim \mathcal{O}(1)$. To the best of our knowledge, no model in the market does this. We believe that, for the time being, the present value of $r$ must be considered an input parameter much 
in the same way as the current value of the Hubble function, $H_{0}$, the temperature of the $\mathrm{CMB}$, the age of the universe, or the ratio between the number of baryons and photons, are -only that the four latter are not laden with any puzzle.

\section{COMPARISON WITH OBSERVATIONAL DATA}

We wish to explore whether the interacting, models alpha, beta, and eta, are consistent with the results of Daly et al. [22]. These are based in the determination of the dimensionless coordinate distance, $y(z)=H_{0}\left(a_{0} \tilde{r}\right)$, and their two first derivatives with respect to redshift, of supernovae type Ia (SN Ia) and radio-galaxies (RG) in the redshift interval $0<z<1.8$. The supernovae samples contains 182 SN Ia of Riess et al. [30], 192 of Davis et al. [31] and 115 of Astier et al. [32] though there is some overlap between the different samples. The RG sample comprehend 19 of Daly et al. [33] plus 11 additional RG.

It is to be stressed that the determination of the dimensionless coordinate distances does not involve any cosmological model, just the Friedmann-Robertson-Walker (FRW) metric. The first and second derivatives of the dimensionless coordinate distance with respect to redshift can be used to build a model-independent determination of the dimensionless expansion rate, $E(z)=H(z) / H_{0}$, and the deceleration parameter, $q(z)=-\ddot{a} /\left(a H^{2}\right)$, see [34].

Indeed, in virtue of the relation between redshift $z$ and the scale factor $1+z=a_{0} / a(t)$, the definition of the Hubble rate, $H=\dot{a} / a$, and the relationship $d t=-a(t) d \tilde{r}$, valid in spatially flat FRW spacetimes for photons flying from their source to the observer, one follows:

$$
E(z)=\frac{H(z)}{H_{0}}=\frac{1}{y^{\prime}(z)},
$$

where a prime indicates derivation with respect to $z$. From last expression the data $y(z)$ can be used to empirically determine $y^{\prime}(z)$ and $E(z)$. Differentiating Eq.(10) with respect to time and using the definition of $q(z)$ leads to

$$
q(z)=-1-(1+z) \frac{y^{\prime \prime}}{y^{\prime}} .
$$

Likewise, differentiating Eq.(3) with respect to time and using Eqs.(10) and (11) produces

$$
P_{\phi}(z)=P_{\phi}(z=0)\left(\frac{E^{2}(z)}{E^{2}(z=0)}\right)\left[\frac{2 q(z)-1}{2 q(z=0)-1}\right] .
$$


Thus, the dimensionless quantities $E(z), q(z)$ and $P_{\phi}(z)$ can be constructed directly from $y^{\prime}$ and $y^{\prime \prime}[34]$.

In virtue of Eqs.(1),(3i), (10) and (11) we get

$$
y^{\prime}(z)=\frac{1}{\left[\Omega_{m 0} \Im_{1}+\Omega_{\phi 0} \Im_{2}\right]^{1 / 2}},
$$

and

$$
y^{\prime \prime}(z)=-\frac{3}{2} \frac{y^{\prime}}{(1+z)}\left[\frac{\Omega_{m 0} \Im_{1}+\Omega_{\phi 0}(1+w) \Im_{2}}{\Omega_{m 0} \Im_{1}+\Omega_{\phi 0} \Im_{2}}\right] .
$$

These expressions are valid for all three models of above. Here $\Omega_{m 0}=\frac{\kappa^{2} \rho_{m 0}}{3 H^{2}}$ and $\Omega_{\phi 0}=$ $\frac{\kappa^{2} \rho_{\phi 0}}{3 H^{2}}$ denote the present day values of the density parameters of DM and DE, respectively. Numerical integration of Eq. (13) yields the dimensionless coordinate distance, $y(z)=$ $\int_{0}^{z} y^{\prime}(z) d z$. In doing this, $w=$ constant is assumed.

Next, we consider the three expressions for the source term $Q$ introduced above.

(i) For model alpha (interaction term $Q=3 \alpha H\left(\rho_{\phi}+\rho_{m}\right)$ ), we find that

$$
\Im_{1}(z)=(1+z)^{n}, \quad \text { and } \quad \Im_{2}=(1+z)^{n+m},
$$

with $n=\frac{3}{2}[2+w-\sqrt{w(w+4 \alpha)}]$, and $m=3 \sqrt{w(w+4 \alpha)}$.

(ii) For model beta $\left(Q=3 \beta H \rho_{m}\right)$ we obtain

$$
\Im_{1}(z)=(1+z)^{n}, \quad \text { and } \quad \Im_{2}=(1+z)^{3(1+w)}-\frac{\beta}{(\beta+w)} \frac{\Omega_{m}}{\Omega_{\phi}} \Im_{1}(z),
$$

with $n=3(1-\beta)$.

(iii) Likewise, for model eta $\left(Q=3 \eta H \rho_{\phi}\right)$

$$
\Im_{1}(z)=(1+z)^{3}-\frac{\Omega_{\phi}}{\Omega_{m}} \frac{\eta}{(\eta+w)} \Im_{2}(z) \text { and } \Im_{2}=(1+z)^{3(1+w+\eta)} .
$$

In the absence of interaction expressions (15), (16), and (17) reduce to Eqs. (1) and (2) of Daly et al. [22].

The evolution of the quantities $y, y^{\prime}$ and $y^{\prime \prime}$ in terms of $z$ are depicted in Figs. 3 through 5. for the three models. We have used different values of the parameters $w, \alpha, \beta$, and $\eta$, and assumed the standard values $\Omega_{m 0}=0.3$ and $\Omega_{\phi 0}=0.7$. Also, the said evolutions of $y$, $y^{\prime}$ and $y^{\prime \prime}$ are compared with the flat $\Lambda \mathrm{CDM}$ model with the quoted values of $\Omega_{m 0}$ and $\Omega_{\phi 0}$.

The data and analysis from the values of $y(z), y^{\prime}(z)$ and $y^{\prime \prime}(z)$ are used to obtain $E(z)$, $q(z)$ and $P_{\phi}(z) / P_{\phi}(z=0)$. The results are shown in Figs. [6 through 8 and, again, contrasted with the standard $\Lambda \mathrm{CDM}$ model. 
Comparison of Figs. 3 through 7 with the corresponding figures of Ref. [22] reveals that the three models, alpha, beta, and eta, are consistent with the analysis of Daly et al. which is based on different samples of SN Ia and 30 RG. It is worthy of mention that, except for adopting the Friedmann-Robertson-Walker metric, the study is independent of any cosmological assumption. We, therefore, conclude this section by stressing that the three models are consistent with the known properties of dark energy.

\section{A STRATEGY TO SOLVE THE COINCIDENCE PROBLEM}

As mentioned above, a cosmological model that predicts a constant or slowly evolving ratio $r$ at late times certainly alleviates the coincidence problem but this is not enough. The model also must predict that $r_{0} \sim \mathcal{O}(1)$. Our strategy is very simple; it rests on the natural assumption that $r(z)$ and $H(z)$ must be closely interrelated. Therefore, if one express $r$ in terms of $H$ the problem of explaining why $r_{0}$ is of order unity reduces to the less pressing

problem of explaining the present value of $H$. This is interesting because while we are not aware of model-independent data of $r$ at different redshifts, two sets of observational data values -though scarce- of $H$ at various redshifts are now available. One data set was compiled by computing differential ages of passively evolving galaxies in the redshift range $0.1<z<1.8$ [35]. The other data set is based on measurements of supernovae type Ia (SN Ia) and 30 radio galaxies up to redshift 1.2 [22].

To express $r$ as a function of $H$ we use the identity $\dot{r}=\dot{H} d r / d H$, with

$$
\dot{H}=-\frac{\kappa^{2}}{2}\left(\rho_{m}+\rho_{\phi}+P_{\phi}\right)=-\frac{3}{2} \frac{1+w+r}{1+r} H^{2},
$$

in Eq. (2). Then, the latter becomes

$$
\frac{d r}{d H}=\frac{\Im}{H}
$$

where

$$
\Im=-2 r \frac{1+r}{1+w+r}\left[w+\frac{\kappa^{2} Q}{9 H^{3}} \frac{(r+1)^{2}}{r}\right] .
$$

Because of we expect $d r / d H>0$ (a very reasonable assumption since $H\left(t<t_{0}\right)>H_{0}$ as observation tells us) the inequality

$$
|w|>\frac{\kappa^{2} Q}{9 H^{3}} \frac{(r+1)^{2}}{r}
$$


is to be satisfied.

We note in passing that for the particular case of the $\Lambda$ CDM model (i.e., $w=-1$ and $Q=0)$ one has $H=H_{0}\left[(1+r) /\left(1+r_{0}\right)\right]^{1 / 2}$.

\section{A. Model alpha}

For this model, Eq. (20) reduces to

$$
\Im=-2 r \frac{1+r}{1+w+r}\left[w+\alpha \frac{(r+1)^{2}}{r}\right],
$$

where we used Eq. (3). The condition given by Eq. (21) leads to restriction $|w|>\alpha(1+$ $r)^{2} / r$.

Using last equation in (19) and integrating, the Hubble function can be expressed in terms of $r$ as

$$
H(r)=H_{0} \exp \left[I(r)-I\left(r_{0}\right)\right]
$$

where $I(r)$ stands for the real part of $\tilde{I}(r)$ with

$$
\tilde{I}(r)=\frac{1}{2} \ln (1+r)-\frac{1}{4} \ln \left[-w r-\alpha(1+r)^{2}\right]-\frac{1}{2} \frac{w+2}{\sqrt{-w(4 \alpha+w)}} \tan ^{-1}\left[\frac{2 \alpha(1+r)+w}{\sqrt{-w(4 \alpha w)}}\right] .
$$

Figure 9 shows the dependence of the Hubble function on the densities ratio for $\alpha=$ $10^{-4}$ and two values of the equation of state parameter, $w$. Likewise, we have plotted the prediction of the $\Lambda \mathrm{CDM}$ model. In all the cases $r_{0}=3 / 7$. For other $\alpha$ values compatible with the restriction $\alpha<2.3 \times 10^{-3}$ set by the Wilkinson Microwave Anisotropy Probe $3 \mathrm{yr}$ experiment the corresponding graphs (not shown) are rather similar.

Unfortunately, as said above, no model-independent data of $r$ at different values of $H$ (or $z$ ) exists whereby, for the time being, we cannot directly contrast this prediction with observation. This is why we turn to determine the dependence of the Hubble factor with redshift and compare it with the two available observational data sets of $H$ vs $z$. The first model-independent data set was obtained from the study of the differential ages of 32 -carefully selected- passively evolving galaxies in the redshift range $0.1<z<1.8$ [35]. The age of each galaxy was found by constraining the age of its older stars with the use of 
synthetic stellar population models. The differential ages roughly yields $d z / d t$, then $H(z)$ is given by $H=-d z /[(1+z) d t]$-see right panel of Fig. 1 in Ref. [35]. The second model-independent data set (see lower panels in Fig. 9 of Daly et al. [22]) was obtained by applying the model-independent analysis method of Ref. [34] to the coordinate distances of 192 SN Ia of Davis et al. [31] and 30 radio galaxies of Ref. [33].

To obtain the expression for $\mathrm{H}(\mathrm{z})$ of this model we combine Eq. (23) with the integral of (2) in terms of $z$ which is

$$
r(z)=\operatorname{Re}\left\{\frac{1}{2 \alpha}\left[-w+\gamma \tan \left(A-\frac{3}{2} \gamma \ln (1+z)\right)\right]\right\}-1,
$$

where Re specifies the real part of the quantity in curly parenthesis, $A=\tan ^{-1}[(w+2 \alpha(1+$ $\left.\left.\left.r_{0}\right)\right) / \gamma\right]$, and $\gamma=\sqrt{-w(w+4 \alpha)}$.

Figures 10 and 11, show the dependence of the Hubble expansion rate on redshift predicted by the model for $\alpha=10^{-4}$ and two values of the equation of state parameter, $w$. For comparison, we have also plotted the prediction of the $\Lambda \mathrm{CDM}$ model. The data in Fig. 10 are borrowed from Simon et al. [35] (full circles) and Daly et al. (full diamonds), and in Fig. 11 from Daly et al. (cf. lower panels of figure 9 in Ref. [22]). As can be seen in both figures, the fit to data does not vary significantly between graphs. Given the scarcity of data it is not worthwhile to compare the $\chi^{2}$ of the different curves.

Figures 9, 10, and 11 taken together suggest that this model may be a solution to the coincidence problem. It fits the available data sets of the evolution of the Hubble factor not less well than the $\Lambda \mathrm{CDM}$ model. Unfortunately, due to the large $1 \sigma$ errors (especially for $z>0.5)$ they cannot discriminate between these two models. A like situation occurs for the beta and eta models detailed below. In consequence, more abundant and accurate observational information about the aforesaid evolution is needed in order decide whether any of the three models can really solve the coincidence problem.

\section{B. Model beta}

This model has been considered in [36] and [37]. Equation (20), with the help of (36), reduces to

$$
\Im=-2 r \frac{1+r}{1+w+r}[w+\beta(r+1)] .
$$

Now, the condition set by Eq.(21) boils down to $|w|>\beta(1+r)$. 
In virtue of Eqs. (19) and (26) the Hubble function can be cast as

$$
H(r)=H_{0} \frac{I(r)}{I\left(r_{0}\right)},
$$

where

$$
I(r)=\sqrt{(1+r)} r^{[-w-1] /[2(\beta+w)]}[\beta(1+r)+w]^{(1-\beta) /[2(\beta+w)]} .
$$

Numerically, the dependence of the Hubble expansion rate upon $r$ is very close to that of model alpha for $\beta$ values similar to $\alpha$. This is why we do not show it here.

The dependence of the Hubble expansion rate on redshift can be obtained by combining Eq. (27) with (9). It is found that the corresponding graphs for $H(z)$ are rather similar to those in Figs. 10 and 11 whereby we do not depict them here.

\section{Model eta}

This model has been studied in [23]. In this instance, Eq.(20) becomes

$$
\Im=-2 r \frac{1+r}{1+w+r}\left[w+\eta \frac{(r+1)}{r}\right],
$$

and the condition set by Eq.(21) simplifies to $|w| r>\eta(1+r)$. With the help of Eqs.(19) and (29) the Hubble function, $H(r)$, is given formally by (27) but this time

$$
I(r)=\sqrt{(1+r)}[\eta+r(w+\eta)]^{-\frac{(1+w+\eta)}{2(w+\eta)}} .
$$

By combining the expression for $H(r)$ with the integral of (2) in terms of $z$,

$$
r(z)=\frac{(1+z)^{-3(w+\eta)}}{(w+\eta)}\left\{r_{0}(w+\eta)+\eta\left(1-(1+z)^{3(w+\eta)}\right)\right\},
$$

one follows $H(z)$. Again, the graphs of $H(z)$ are very close to those of Figs. 10 and 11 and we do not show them here.

\section{DISCUSSION}

Nowadays the leading candidate for dark energy is the popular $\Lambda \mathrm{CDM}$ model. It is simple, it has just one free parameter -the vacuum energy density- and fits reasonably well most (if not all) observational data. However, it presents the puzzle (apparent or not) that we happen to live at a very special epoch: the transient epoch at which the ratio between 
the densities of matter and vacuum energy is of order unity. To solve this one must look for alternative models in which the ratio $r$ stays constant or varies very slowly, around the present time, with respect to the Universe expansion. Interacting models (with $Q>0$, see Eq. (2) ) possess this property. But it does not suffice. The successful model must also predict $r_{0} \sim \mathcal{O}(1)$.

In this paper we considered three interacting models that present the first characteristic and may also have the second one. To decide on the latter one must contrast the $r(z)$ function predicted by each model with observation. Regrettably, as far as we know, modelindependent data about $r(z)$ do not exist. However, albeit with wide uncertainties, there exist model-independent data of the evolution of the Hubble factor up to $z=1.8$ [22, 35]. Therefore, we have numerically determined $H(r)$ and $H(z)$ for each model and compared the latter with these observational data. All three models show compatibility with the data but only in a degree similar to the $\Lambda$ CDM model. Future, more accurate and extensive measurements will likely tell us whether the latter model fits the data better or worse than interacting models (the ones presented in this paper or maybe others). If, in the end, the $\Lambda \mathrm{CDM}$ model makes the best fit, then we may conclude that the coincidence problem is not a problem any more; it is simply a coincidence and therefore not a problem.

\section{Acknowledgments}

S.d.C. was supported from Comisión Nacional de Ciencias y Tecnología (Chile) through FONDECYT Grants No. 1040624, No. 1051086, and No. 1070306 and by the PUCV. R.H. was supported by the "Programa Bicentenario de Ciencia y Tecnología" through the Grant "Inserción de Investigadores Postdoctorales en la Academia" N0 PSD/06. D.P. acknowledges "FONDECYT-Concurso incentivo a la cooperación internacional" No. 7070003, and is grateful to the "Instituto de Física" for warm hospitality; also D.P. research was partially supported by the "Ministerio Español de Educación y Ciencia" under Grant No. FIS2006-12296-C02-01.

[1] V. Sahni, Lect. Notes Phys. 653, 141 (2004); J.A.S. Lima, Braz. J. Phys. 34, 194 (2004);

L. Barnes, M.J. Francis, G.F. Lewis, and E.V. Linder, Publ. Astron. Soc. Australia 22, 315 
(2005); E.J. Copeland, M. Sami, and S. Tsujikawa, Int. J. Mod. Phys. D 15, 1753 (2006); L. Perivolaropoulos, Third Aegean Summer School, The Invisible Universe Dark Matter and Dark Energy, astro-ph/0601014; R. Durrer and R. Maartens, Gen. Relativ. Grav. 40, 301 (2008).

[2] T. Padmanbahn, Phys. Rep. 380, 235 (2003).

[3] A. Coley and P. Bylliard, Phys. Rev. D 61, 083503 (2000); L. Amendola, Phys. Rev. D 62, 043511 (2000); L. Amendola and D. Tocchini-Valentini, Phys. Rev. D 64, 043509 (2001); G. Farrar and P.J.E. Pebles, Astrophys. J. 604, 1 (2004); S. del Campo, R. Herrera and D. Pavón, Phys. Rev. D 70, 043540 (2004); R-G. Cai and A. Wang, J. Cosmology Astropart. Phys. 03(2005)002; B. Wang et al., Phys. Lett. B 624, 141 (2005); D. Pavón and W. Zimdahl, Phys. Lett. B 628, 206 (2005); B. Wang et al., Phys. Lett. B 637, 357 (2006); J.D. Barrow and T. Clifton, Phys. Rev. D 73, 103520 (2006); S. Lee, G.-C. Liu, and K.-W. Ng, Phys. Rev. D 73, 083516 (2006); B. Hu and Y. Ling, Phys. Rev. D 73, 123510 (2006); L. Amendola, M. Quartin, S. Tsujikawa, and I. Waga, Phys. Rev. D 74, 023525 (2006); B. Guberina et al., J. Cosmology Astropart. Phys. 01(2007)012; R. Horvath, Phys. Lett. B 648, 374 (2007); R. Mainini, and S. Bonometto, J. Cosmology Astropart. Phys. 06(2007)020; O. Bertolami, F. Gil Pedro and M. Delliou, Phys. Lett. B 654, 165 (2007); W. Zimdahl, arXiv:0705.2131 [gr-qc]; N. Banerjee, S. Das and K. Ganguly, arXiv:0801.1204 [gr-qc]; C.G. Böhmer, G. Caldera-Cabral, R. Lazkoz, and R. Maartens, Phys. Rev. D. 78, 023505 (2008); R. Bean, E. Flananagan, and M. Trodden, Phys. Rev. D 78, 023009 (2008); K. Karwan, J. Cosmology Astropart. Phys. 05(2008)011; T. Koivisto and D.F. Mota, Astrophys. J. 679, 1 (2008); B.M. Schäfer, Mon. Not. R. Astron. Soc. 388, 1403 (2008); V. Pettorino and C. Baccigalupi, Phys. Rev. D 77, 103003 (2008); L.P. Chimento and M. Forte, Phys. Lett. B 666, 205 (2008); M. Jamil, arXiv:080102.2896.

[4] J.S. Alcañiz and J.A.S. Lima, Phys. Rev. D 72, 063516 (2005).

[5] D. Pavón and B. Wang, Gen. Relativ. Grav. (in the press), arXiv:0712.0565 [gr-qc].

[6] J. Khoury, and A. Weltman, Phys. Rev. Lett. 93, 171104 (2004); ibid 2004, Phys. Rev. D 69, $044026(2004)$.

[7] P.J.E. Peebles and B. Ratra, Rev. Mod. Phys. 75, 559 (2003).

[8] K. Hagiwara, et al., Phys. Rev. D 66, 010001 (2002).

[9] S. del Campo, R. Herrera and D. Pavón, Phys. Rev. D 78, 021302 (2008).

[10] J. Martin, private communication. 
[11] R.-G. Cai and A. Wang, Phys. Rev. D 73, 063005 (2006).

[12] R. Bean and J. Magueijo, Phys. Rev. D 66, 063505 (2002).

[13] L. Amendola, M. Gasperini, and F. Piazza, Phys. Rev. D 74, 127302 (2006).

[14] M. Manera and D.F. Mota, Mon. Not. R. Astron. Soc., 317, 1373 (2006).

[15] N. Tetradis, Phys. Lett. B 362, 463 (2006); N. Brouzakis, and N. Tetradis, J. Cosmology Astropart. Phys. 01(2006)004.

[16] N. Tetradis, J.D. Vergados, and A. Faessler, Phys. Rev. D 75, 023504 (2007).

[17] D. Layzer, Astrophys. J. 138, 174 (1963).

[18] P.J.E., Principles of Physical Cosmology, Princeton University Press (Princeton, N.J., 1993).

[19] E. Abdalla, L.R. Abramo, L. Sodre, and B. Wang, arXiv:0710.1198 [astro-ph].

[20] O. Bertolami, F. Gil Pedro, and M. Le Delliou, Phys. Lett. B 654, 165 (2007).

[21] G. Olivares, F. Atrio-Barandela, and D. Pavón, D. Phys. Rev. D 77, 103520 (2008).

[22] R.A. Daly, S.G. Djorgovski, K.A. Freeman, M. Mory, C.P. O’Dea, P. Kharb, and S. Baum, Astrophys. J. 677, 1 (2008).

[23] J.-H. He and B. Wang, J. Cosmology Astropart. Phys. 06 (2008)010.

[24] G. Olivares, F. Atrio-Barandela, and D. Pavón, Phys. Rev. D 71, 063523 (2005).

[25] G. Olivares, F. Atrio-Barandela, and D. Pavón, Phys. Rev. D 77, 063513 (2008).

[26] W. Zimdahl, D. Pavón, and L.P. Chimento, Phys. Lett. B 521, 133 (2001).

[27] L.P. Chimento, A.S. Jakubi, D. Pavón, and W. Zimdahl, Phys. Rev. D 67, 083513 (2003).

[28] L.P. Chimento, A.S. Jakubi, and D. Pavón, D. Phys. Rev. D

[29] S. del Campo, R. Herrera and D. Pavón, Phys. Rev. D 74, 023501 (2006). 67, 087302 (2003).

[30] A.G. Riess et al., Astrophys. J. 659, 98 (2007).

[31] T.M. Davis et al., Astrophys. J. 666, 716 (2007).

[32] P. Astier et al., Astron. Astrophys. 447, 31 (2006).

[33] R.A. Daly et al., arXiv:0710.5112,

[34] R.A. Daly and S.G. Djorgovski, Astrophys. J. 597, 9 (2003).

[35] J. Simon, L. Verde and R. Jiménez, Phys. Rev. D 71, 123001 (2005).

[36] Z.K. Guo, N. Ohta, and S. Tsujikawa, Phys. Rev. D 76, 023508 (2007).

[37] L. Amendola, G. Campos, and R. Rosenfeld, astro-ph/0610806. 

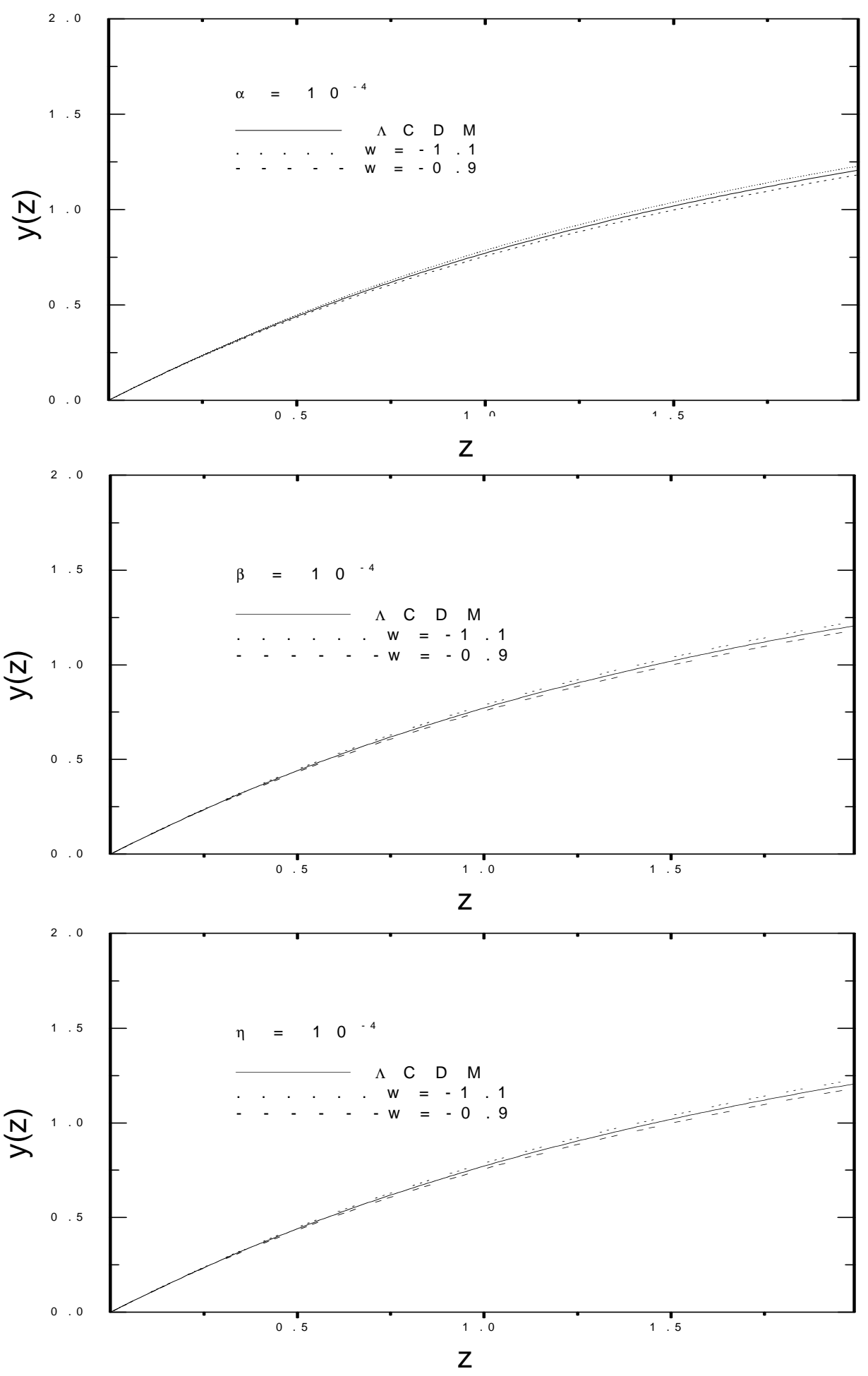

FIG. 3: From top to bottom, dimensionless coordinate distance, $y(z)=H_{0}\left(a_{0} \tilde{r}\right)$, in terms of the redshift for models alpha, beta, and eta. For comparison, the prediction of the $\Lambda$ CDM model is also shown. 

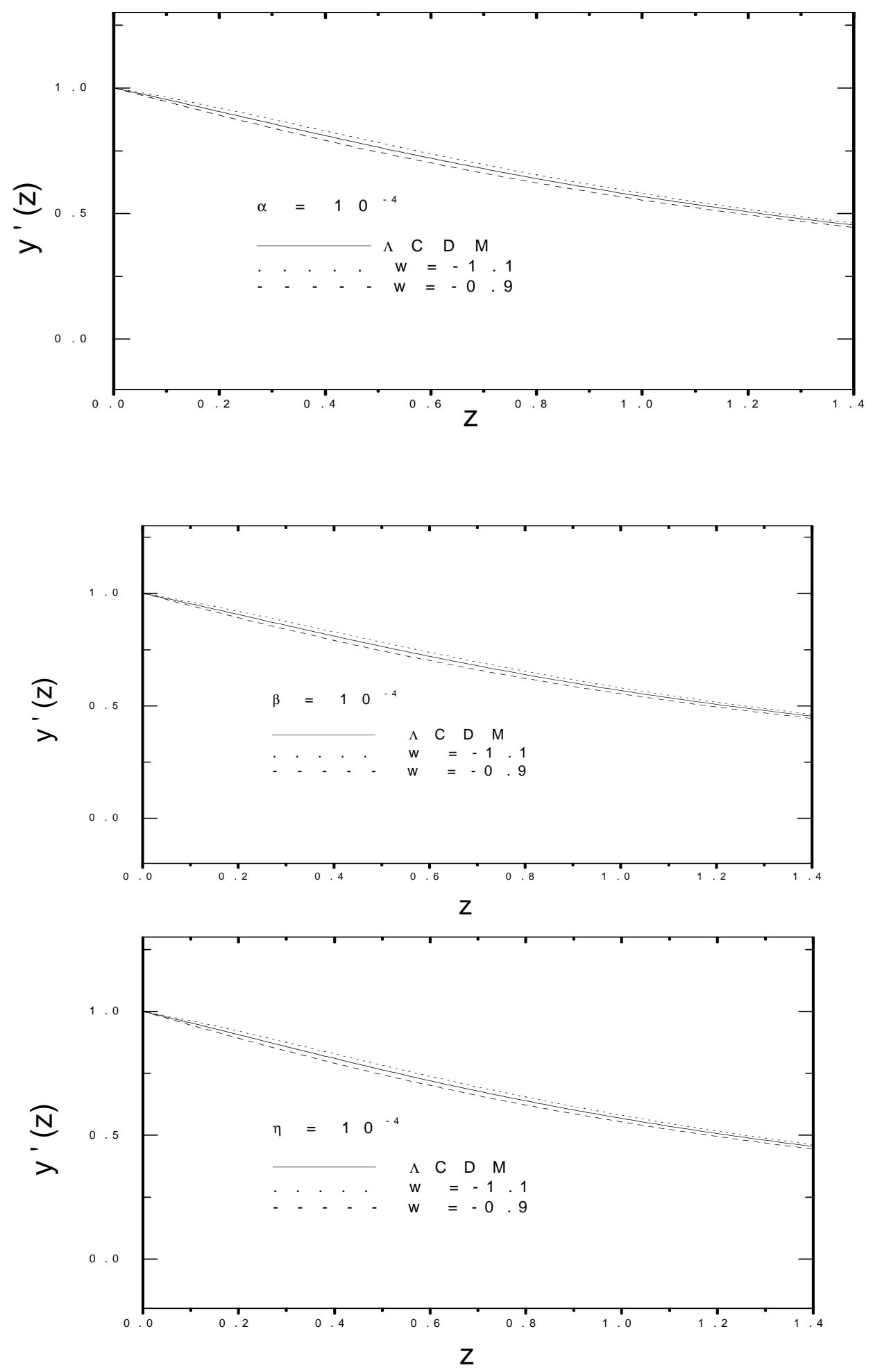

FIG. 4: From top to bottom, first derivative of the dimensionless coordinate distance in terms of redshift for models alpha, beta, and eta. For comparison, the prediction of the $\Lambda$ CDM model is also shown. 

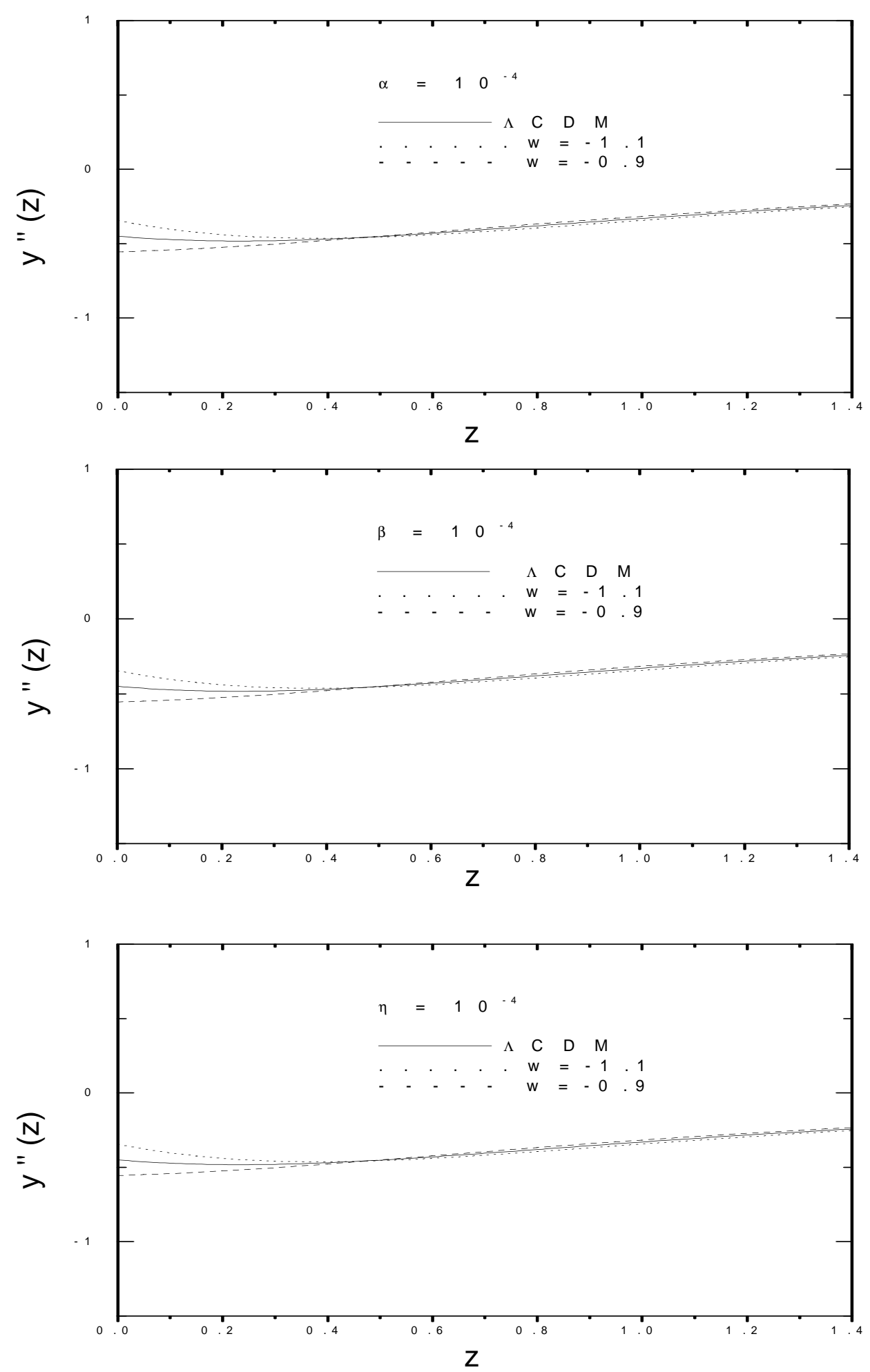

FIG. 5: From top to bottom, second derivative of the dimensionless coordinate distance in terms of redshift for models alpha, beta, and eta. For comparison, the prediction of the $\Lambda$ CDM model is also shown. 

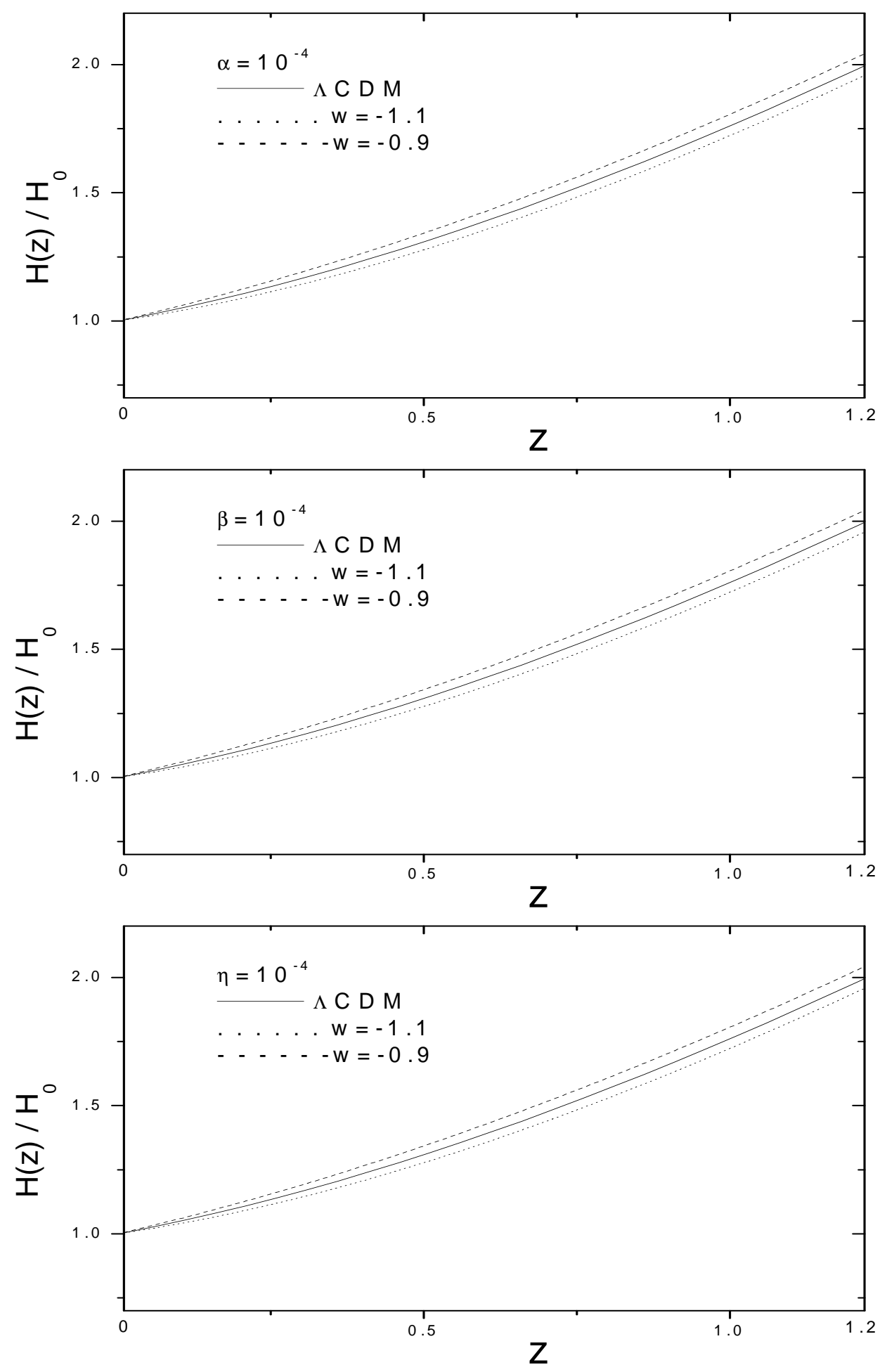

FIG. 6: From top to bottom, evolution of the Hubble factor for models alpha, beta, and eta. For comparison, the prediction of the $\Lambda \mathrm{CDM}$ model is also shown. 

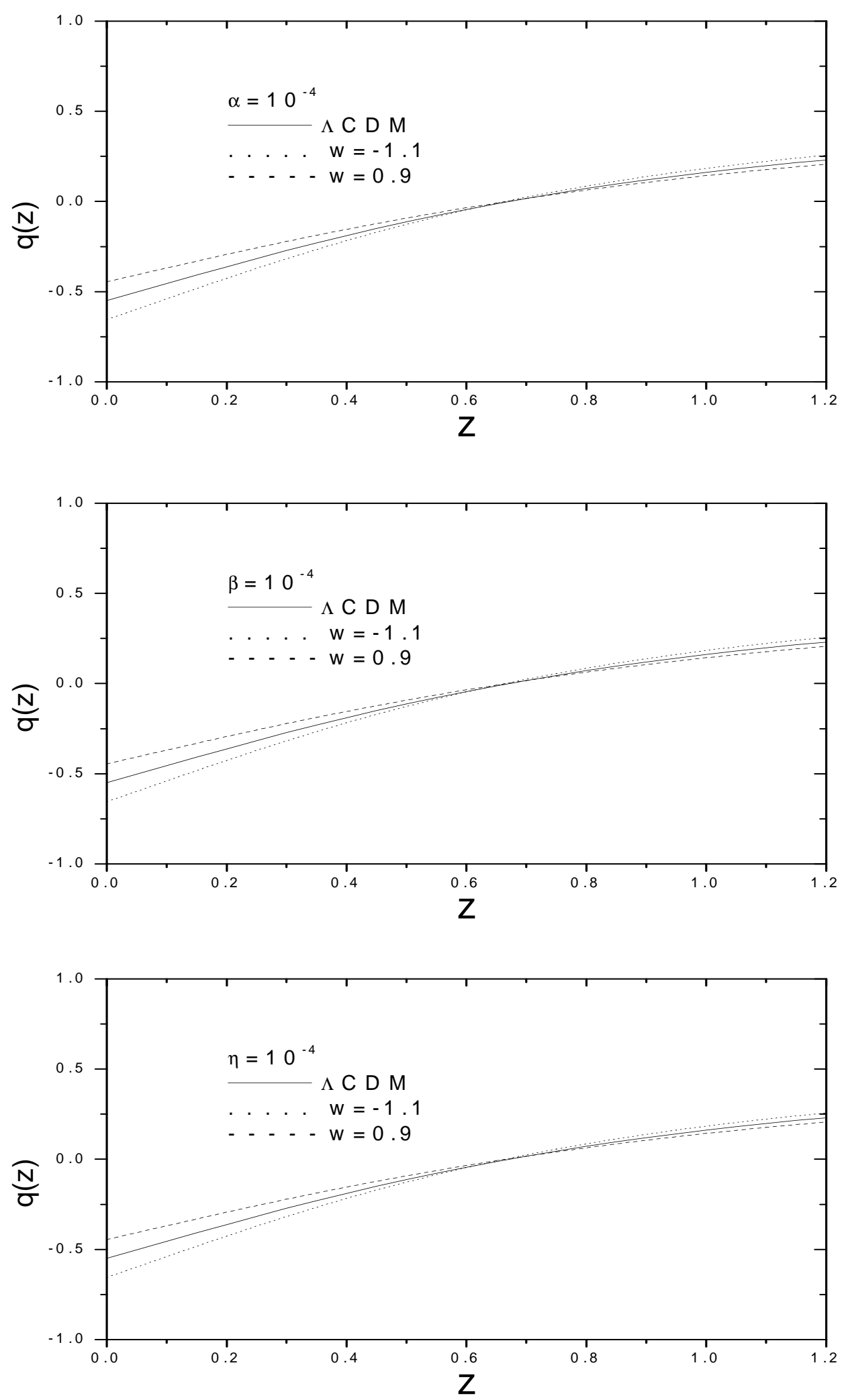

FIG. 7: From top to bottom, evolution of the deceleration parameter for models alpha, beta, and eta. For comparison, the prediction of the $\Lambda \mathrm{CDM}$ model is also shown. 

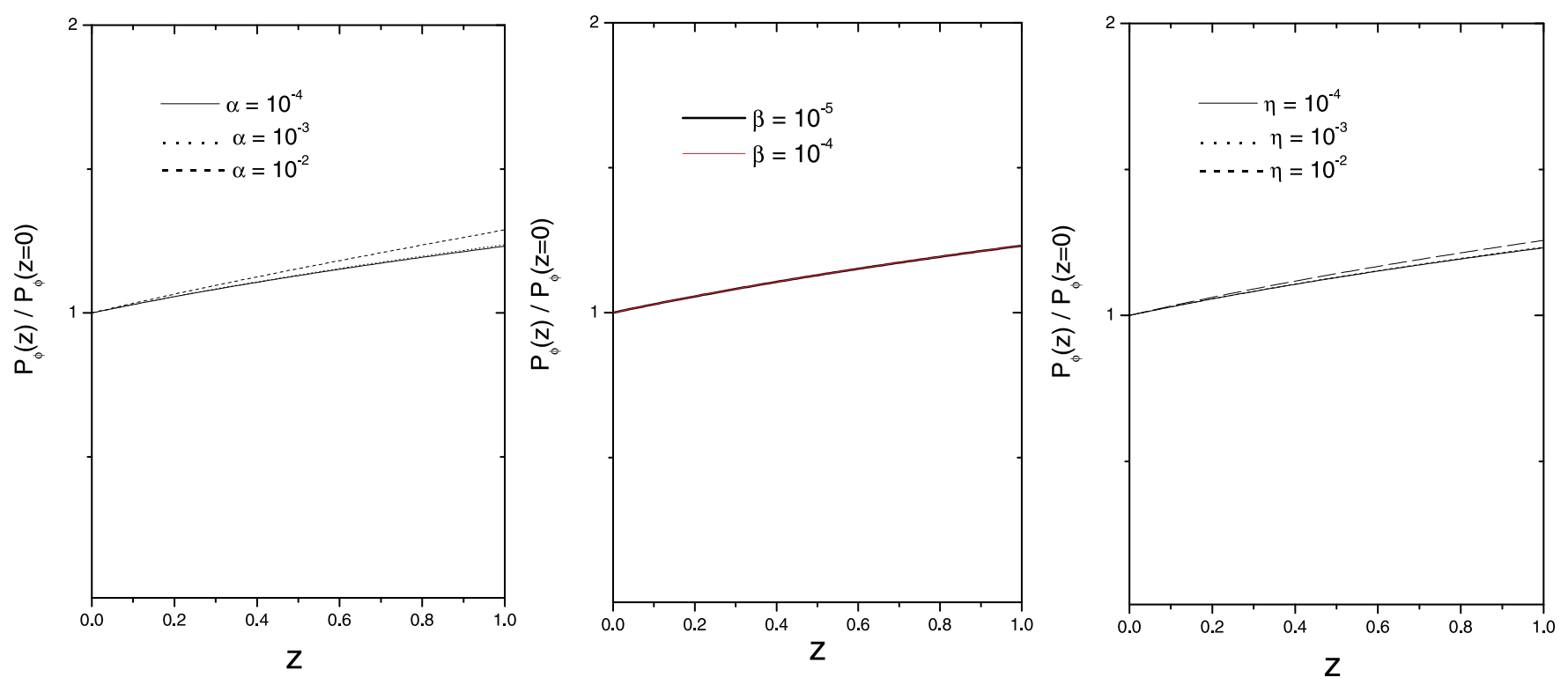

FIG. 8: From left to right, evolution of the ratio $P_{\phi}(z) / P_{\phi}(z=0)$ with redshift for models alpha, beta, and eta. 


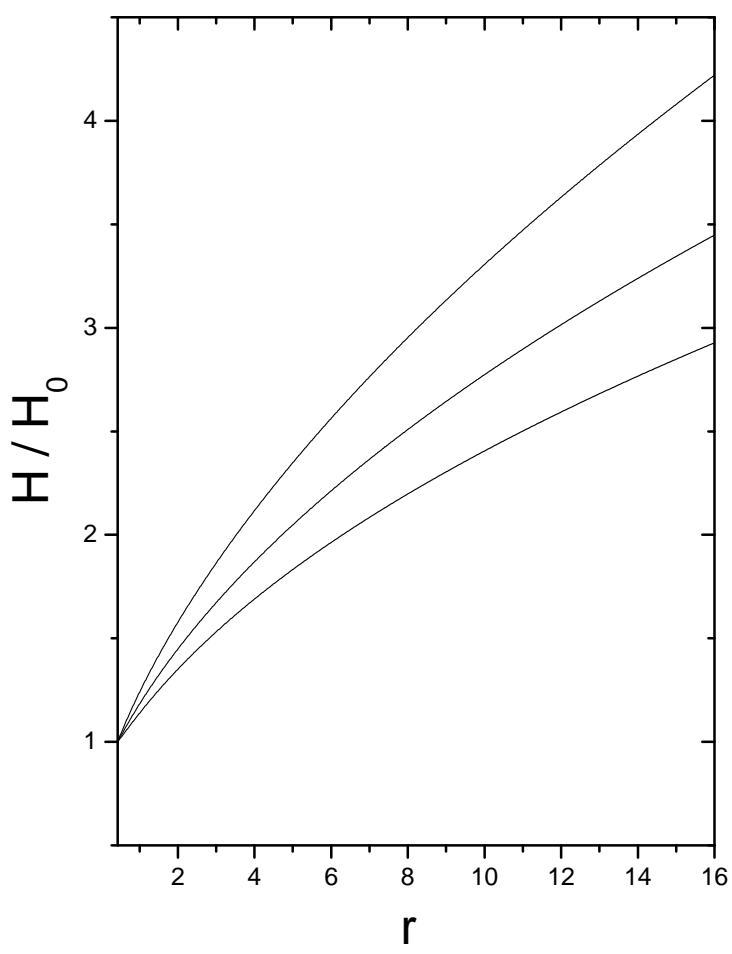

FIG. 9: Evolution $H$ vs the ratio $r=\rho_{m} / \rho_{\phi}$ as given by Eq. (23) -model alpha- with $\alpha=10^{-4}$ for $w=-0.9$ (quintessence, top line) and $w=-1.1$ (phantom, bottom line). Also shown is the prediction of the $\Lambda$ CDM model (middle line). In drawing the curves we have set $r_{0}=3 / 7$. 


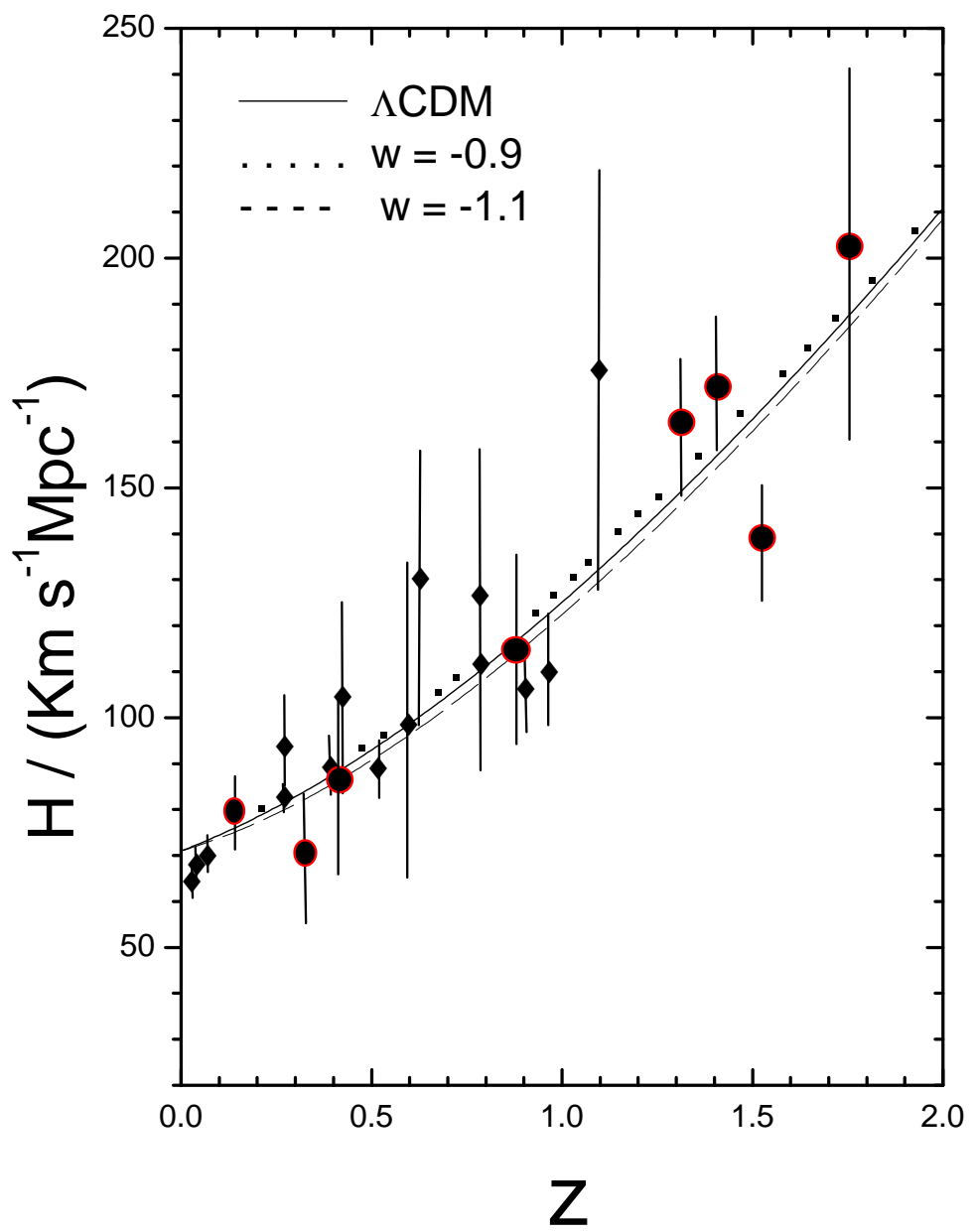

FIG. 10: Evolution $H$ vs $z$ with $\alpha=10^{-4}$ for $w=-0.9$ (quintessence) and $w=-1.1$ (phantom). Also shown is the prediction of the $\Lambda$ CDM model (solid line). In all the cases we have fixed $r_{0}=3 / 7$ and $H_{0}=71 \mathrm{~km} / \mathrm{s} / \mathrm{Mpc}$. The data points with their $1 \sigma$ error bars are borrowed from Simon et al. Ref. [35] (full circles) and table 2 of Ref. [22] (full diamonds). 


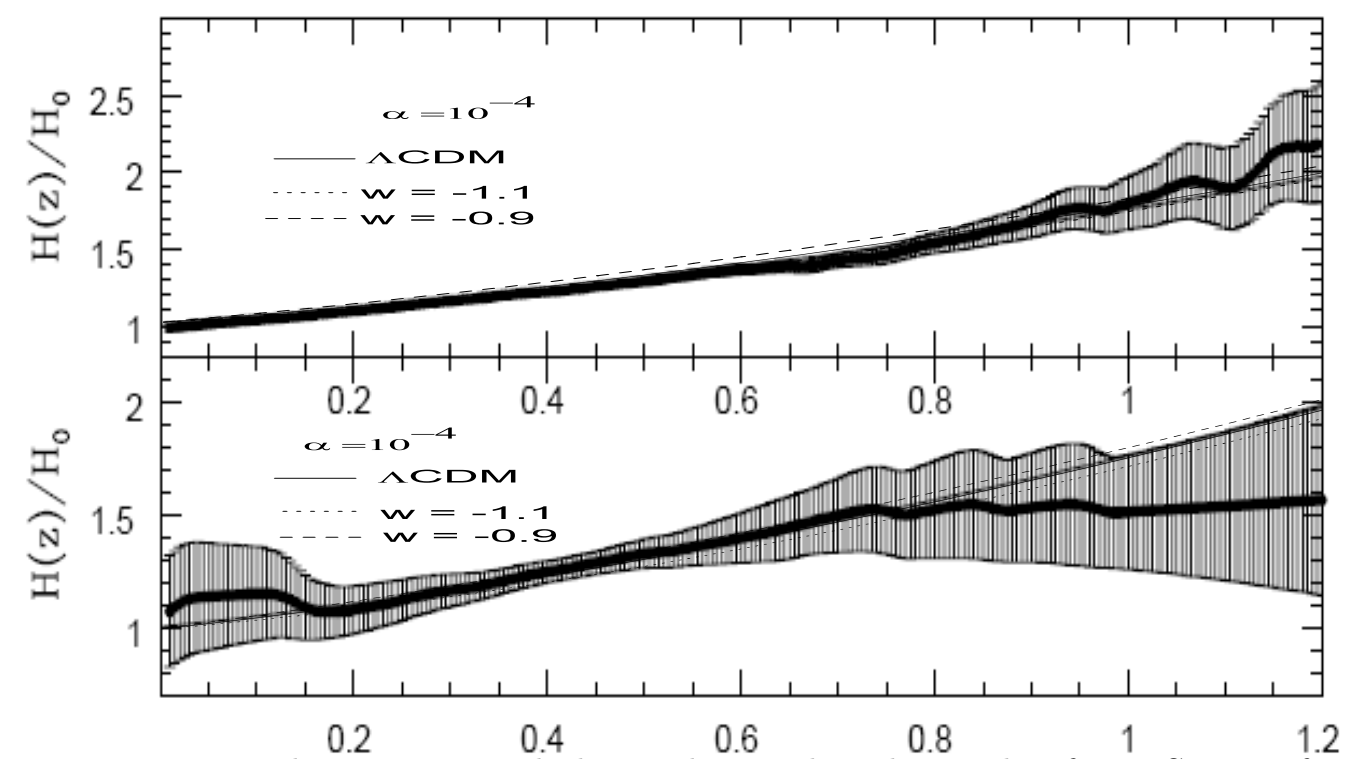

FIG. 11: Same as Fig. 10, The upper panel shows the combined sample of 132 SN Ia of Ref. [31] and 30 radio galaxies of Ref. [33]. The bottom panel shows the 30 radio galaxies only. The data points with their $1 \sigma$ error intervals and the best-fit curve (big solid line) in both panels are borrowed from Daly et al., Ref. [22]. 